Rapid Reviews COVID-19 •

\title{
Review 1: "A Prenylated \\ dsRNA Sensor Protects \\ Against Severe COVID-19 \\ and is Absent in Horseshoe \\ Bats"
}

\author{
Margo Brinton 1 \\ ${ }^{1}$ Georgia State University, Biology Dept., 623 PSC 161 Jesse Hill Jr Drive, Atlanta, Georgia
}

Published on: Sep 16, 2021

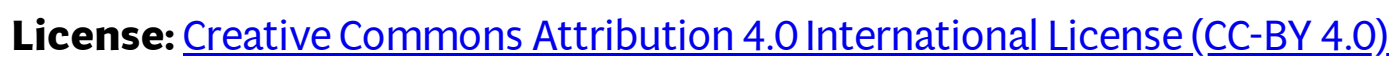




\section{$\underline{\text { RR:C19 Evidence Scale rating by reviewer: }}$}

- Reliable. The main study claims are generally justified by its methods and data. The results and conclusions are likely to be similar to the hypothetical ideal study. There are some minor caveats or limitations, but they would/do not change the major claims of the study. The study provides sufficient strength of evidence on its own that its main claims should be considered actionable, with some room for future revision.

$* * * * * * * * * * * * * * * * * * * * * * * * * * * * * * * * * * * * * * *$

\section{Review:}

The scope of this study is broad and most of the claims are justified by the data. Previous screens as well as ones performed by the authors of this study identified OAS1 as an interferon-inducible inhibitor of a SARS-CoV-2 infection. The authors then showed that the p46 isoform of human OAS1 has strong antiviral activity against a SARS-CoV-2 infection through the canonical RNase L pathway and that the presence of the p46 C-terminal prenylation site is required for this activity using multiple experimental strategies in cell cultures stably expressing variant $\mathrm{p} 46$ proteins. Previous studies showed that human OAS3 has antiviral activity against other coronaviruses and that these viruses encode 2 ' -5 ' phosphodiesterases to counteract OAS activity. The authors searched for phosphodiesterase genes in coronavirus sequences and found that none of the known coronaviruses infecting Rhinolephoidea, which includes the SARS viruses, encoded a phosphodiesterase. They also analyzed host OAS1 gene sequences and found that the prenylation site region of the OAS1 gene sequence had been deleted in the genomes of all bats in the Rhinolophoidea superfamily due to ancient retrotransposition and confirmed that the bat OAS1 protein did not have anti-SARS-2-CoV activity. These data provide an explanation for why coronaviruses that evolved in these bat species are susceptible to a prenylated OAS1 isoform and do not encode a phosphodiesterase. However, bats encode an OAS3 gene but its presence and antiviral activity were not analyzed.

Increased OAS1 expression (p46 could not be assessed) was identified in lung tissue of deceased COVID-19 patients, indicating interferon induction. A single blood sample from 499 hospitalized COVID-19 patients with mild or severe disease was also analyzed by RNAseq. This analysis allowed the determination of the patients' OAS1 SNP genotype as well as the relative expression level of the different OAS1 mRNAs in 
the blood. Although a higher number of individuals expressing p46 were found in the mild disease group, there were also many with severe disease who expressed p46.

The data in this manuscript identifies prenylated OAS1 p46 as a robust antiviral host factor for SARS-2-CoV. Further, OAS1 p46 antiviral activity is expected to reduce virus replication in infected individuals. However, OAS1 is an interferon-stimulated gene, and the extent, timing, and location of interferon production in individual patients would determine the relative benefit of OAS1 p46 in providing protection from severe disease. Also, human susceptibility factors could override the effect of $\mathrm{p} 46$.

The statements suggesting that patients of African descent would be more resistant to severe COVID-19 disease are not consistent with clinical data. 